\title{
TOPOLOGY TOOLS FOR NETWORK ANALYSIS ON GIS PLATFORMS
}

\author{
C. Agüero, G. di Mauro, J. Vignerte, D. Anaut, J. Strack \\ Departamento de Ing. Eléctrica, Universidad Nacional de Mar del Plata, Argentina.
}

\begin{abstract}
Vector Geographic Information Systems (GIS) have network analysis tools that are of great importance in the modeling of electrical networks and especially for any study based on the interconnectivity of their sections. This work presents the development of computer tools that use as a basis the analysis of network topologies and their application for the detection of areas interconnected or affected by interruptions in supply, the identification and selection of each of the elements represented and the possibility of exchanging data with other calculation software.
\end{abstract}

Keywords: Software, GIS, modeling, network analysis, exchanging data, power quality, geographic information system

Cite this Article: C. Agüero, G. di Mauro, J. Vignerte, D. Anaut, J. Strack, Topology Tools for Network Analysis on GIS Platforms, International Journal of Electrical Engineering \& Technology, 10(6), 2019, pp. 12-20.

http://iaeme.com/Home/issue/IJEET?Volume $=10 \&$ Issue $=6$

\section{INTRODUCTION}

Electric power distribution companies use their grids daily as a base tool for planning, operating and maintaining them.

Energy companies use the computational tools for geoprocessing, called Geographic Information Systems (GIS), and the ability to query and analyze information directly from the georeferenced database for use in constructed models allows you to perform more refined studies and a higher level of detail This integration between the georeferenced database with the models proposed in the methodology is considered, making planning possible to be done from the data from the company's georeferenced information system (GIS) database. [1]

Geographic Information Systems which have tools for the generation and maintenance of topologies and these are the basis for the development of the analysis tools presented in this work. Topology is the field of mathematics that studies the relationships of elements in space. "The topology of a map is the set of relationships that describe the relative position of its components" [2].

The conception of these relationships varies between Raster and Vector Geographic Information Systems. In Raster (matrix) systems, relationships occur between cells as generally neighborhood analysis, conforming spatial features from physical proximity and attributes between pixels. Vector systems are typically based on an arc-node topology that is 
defined by directionality, connectivity, and proximity between vectors; so that from these and other values the different spatial features are defined.

Topology is of great importance in the development and evolution of GIS. It is decisive in its analytical capabilities and largely defines the development of geographic information formats. [3]

There are basically three types of topologies [4]:

Node Topology-Composed of a system of related point objects that can be used to analyze links between individual points on a map, such as substations or transformation centers. Nodes have only X, Y location and do not occupy area.

Network Topology: where a connected link system can be used to develop analysis on linear survey features such as power lines. A wide spectrum of alternatives is added by assigning a direction and weight value to segments in a hopological network.

Polygon Topology: Generated by areas, or polygons, these are formed of interconnected segments (links). Polygon topologies can be used to analyze relationships between land surfaces.

\section{METHODOLOGICAL APPROACH}

In this work we focus on the use of network topologies and in particular their application to continuity analysis. This is of great interest to the Elective Energy Distribution Companies allowing to model and simulate operations in the networks, identify interconnected sections, detect areas affected by interruptions in the supply of electricity, identification of circuits and customers supplied.

In a Vector GIS, a network is an interconnected system of linear elements, which form a spatial structure through which flows of some kind can pass: people, energy, etc. [4] A GIS allows to analyze a network from different points of view, being the most used to search for optimal routes and locate services. [5]

It is decided to use Autodesk company AutoCAD Map software as a GIS tool. It is considered convenient by several factors; Among the most important are the fact that multiple programming and customization tools are available that allow to automate tasks and fundamentally because in the technical areas of distributors are familiar with the working environment of CAD programs for being common use for the management of graphical information. AutoCAD Map is based on AutoCAD, and from it inherits most of the virtues of its editing engine. This makes it easy to learn and leverage the information available along with the possibility of using the same work environment for digitization, editing, visualization and printing.

The GIS has tools for creating, maintaining, and analyzing topologies that are of vital importance in the studies that are developed in this work.

\subsection{The Preparation of the Map}

Before you create a topology, you must verify that the drawing information does not contain errors in the geometry such as lines that do not touch or overlap without intersection. Care in digitization and in the case of existing drawings is essential to use cleaning tools to debug information that is intended to be used in topologies.

Information with duplicate lines, intersecting lines, short lines, will cause the topology creation process to fail. Any of the following issues causes the network or polygon topology to fail to create, incomplete or incorrect.

- Insufficient Objects - Objects within one tolerance to another. 
- Loose Objects - Objects with at least one endpoint that is not shared with another object.

- Cut Objects - Objects that intersect each other without an intersection node

- Clustered Nodes - Nodes that are within a tolerance of each other.

- Duplicate Objects - Objects that share the same start point and endpoint.

- Short Objects - Objects that are shorter in length than the specified tolerance

Cut objects must be carefully controlled; some objects should not cut at intersections, for example, segments that represent network sections that intersect but do not connect.

In the proposed methodology for the representation of network legs is used the polyline, this allows to integrate into a feature more than one leg facilitating the selection in data extraction or editing tasks.

At the ends of the segments there are nodes. Segments start and end with nodes, and nodes allow joining between two or more segments. In AutoCAD Map, network topologies can be created from polylines, but only after line integration has been verified. If Grouped Nodes, Duplicate Lines, Insufficient Objects are found in the network geometry, they must be removed before proceeding with topology creation.

To avoid confusion, a filled circle block was created that is located at the junctions of lines that join, while it does not play a role in creating topologies, it facilitates visual review to confirm that there is indeed a join between network sections (Fig. 1).

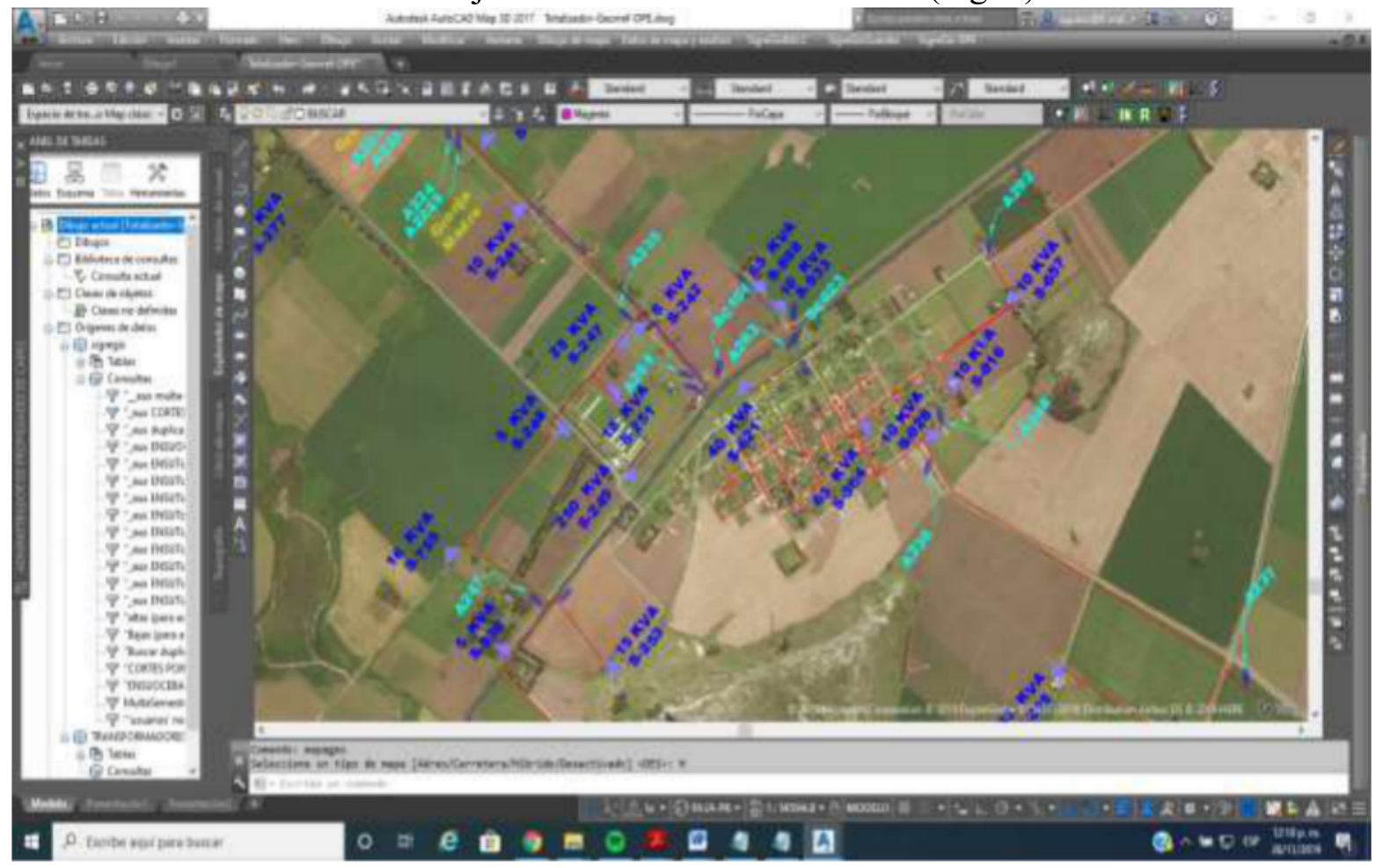

Figure 1. Network Representation

Once the map has the geometry of the network, it is possible to add intelligence by creating a network topology, which can be consulted for information, used to create thematic maps, or to run analyses such as Optimal Paths and Track Flows, in the latter case are derived numerous applications of interest for the analysis of electrical continuity.

Each network topology creates an object data table that stores the information for each segment (Link). Fields include the Segment Identification Number, The Initial node and end 
node identification number, the Associated Address Value, and the Direct and Reverse Resistance Values.

In a network topology, direction values identify the sense that movement can take across segments. All moves are relative to the start and end node that are created with the topology. With each segment in the network topology, one of three possible address values are associated. For a value of 0 , movement is possible in both directions. For a value of 1 , the movement is possible in the same direction in which the segment was created. For a value of -1 , it is possible to move in the opposite direction of the direction in which the segment was created. In electrical networks this is of importance in high voltage to medium voltage or medium voltage to low voltage recesses where the circulation of energy is only possible from higher to lower voltage level.

Resistance values represent the amount of force required for movement to occur through a segment. Direct Resistance is the value associated with the movement in the direction that the segment was created. Reverse Resistance is the value associated with movement in the opposite direction as seen in Table I.

Table 1 Network Topology Information

\begin{tabular}{|l|c|}
\hline \multicolumn{1}{|c|}{ Name of Topology } & Fases \\
\hline Type & Link \\
\hline ID & 110240 \\
\hline Initial Node & 560886 \\
\hline End Node & 55923 \\
\hline Direction & 0 (Bidireccional) \\
\hline Direct Resistance & 243.55 \\
\hline Reverse Resistence & 243.55 \\
\hline
\end{tabular}

The electrical network is represented by polylines with associated object data (fig. 2). Once a network topology has been created, you can start using it to develop the analysis.

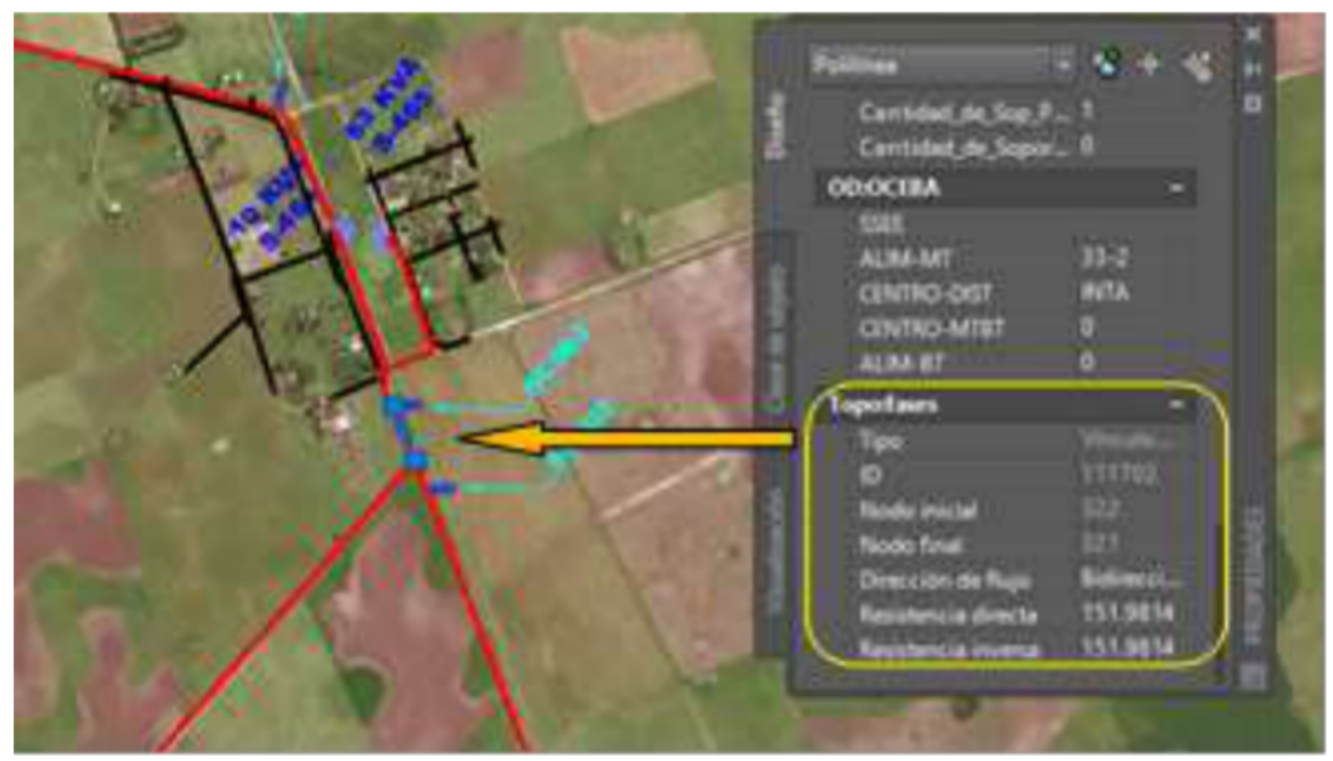

Figure 2. Network Attributes

\subsection{Flood Trace}

Tracking a Flow results in all nodes and segments within the specified radius from the defined point. The GIS can display the result as a preview by altering the color of all segments along paths within a specified resistance range or generating a new topology and thus using the 
result in subsequent analyses by identifying, selecting, and/or exporting data from the entities involved to specific computation software [6]. This type of analysis is very useful for displaying the network sections affected by an outage if a high convenient value is assigned to the Maximum Resistance parameter and the resistance offered by the maneuvering and protection equipment involved.

\section{RESULTS}

\subsection{Creating and Maintaining the Network Topology}

One of the premises was to simplify system maintenance and upgrade to the maximum. A tool was developed that summarizes in a single command all the processes of creating upgrade or editing the network topology. This command was called REMAKETOPOLOGY

Running this command performs the following steps: Select the required features; you must select all energized items in the High, Medium, and Low Voltage network until you reach the client. In order to automate this selection of features to be taken into account in the generation of the topology it is necessary to respect the layer assignment established by the methodology and the use of a library provided with blocks for equipment

.Check the status of maneuvering and protection equipment: the proposed symbology for this equipment is designed so that the open or closed state is clearly displayed on the printed planes and simultaneously depending on their status the tool assigns zero resistance to closed and high open disconnectors.

Assign direction to network legs in HV-MV and MV-LV recesses: in the representation of the Transformer Stations are incorporated sections that are recognized and is assigned directionality avoiding the flow of current for the network legs in HV-MV and MV-LV recesses preventing a low voltage continuity analysis extends to medium voltage or medium voltage to high voltage.

For network upgrade and maintenance, incorporation of partner highs and lows, or circuit modifications must follow the pre-detailed cleanup rules, supported feature type, and layer where each item is located.

\subsection{Development of Tools for Disconnector}

A tool (ONOFF command) was developed that simplifies in a single step the tasks of editing the resistance value of the maneuvering equipment and the protection of the network and its visualization.

Requests to select a disconnector block and simultaneously toggles the state, both in the visual identification of the symbol and in the resistance assignment within the topology.

In AutoCad Map to perform an edit within a topology, it must be created and active, so the command verifies the state of the network topology and if necessary creates it by invoking the REMAKETOPOLOGY detailed at the previous point.

The command asks to select a disconnector block (Fig 3)

- Checks its status from the open or closed attribute

- Toggles the state

- Assigns resistance value. 


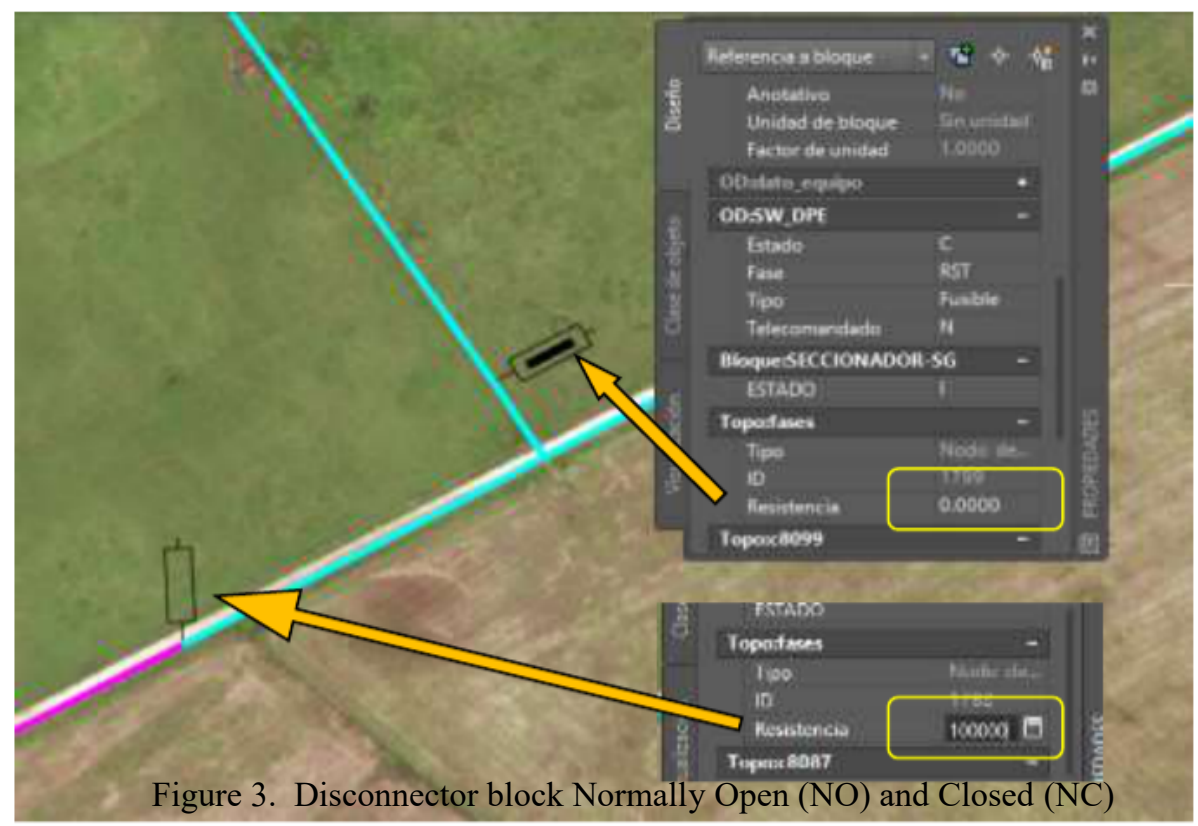

Figure 3 Disconnector block Normally Open (NO) and Closed (NC)

\subsection{Topology Testing Tool}

This tool performs a flow trace analysis in preview mode to check the continuity of the network from a point indicated on the screen.

Displays all possible paths from a selected node and adds the resistance value for each of the links and the nodes they pass through. Path tracking stops when the sum reaches the maximum crawl resistance value, in the case of power grids it will stop at a network end, open disconnector, or transformer station represented by a run with resistance infinite in the reverse direction to the energy flow.

The operation of the command (TESTTOPOLOGIA) is detailed below:

- Verify that it is created, active, and selects the topology.

- Asks to indicate a point on a network leg, identifies the nearest node in the topology, and sets it as the Initial Node

- Assigns as Tracking Parameters, Maximum Resistance Value 100000, a value high enough to ensure that the analysis is extended in all electrically interconnected sections. This parameter means that Flow Tracking travels a maximum of 100000 units before finishing. This value includes the resistance of the networks, which is their length by default, and the resistance of the disconnectors. If a disconnector is closed, the resistor is zero, open greater than 100000 .

- The command takes into account the addressed legs assigned to the network legs in HV-MV and MV-LV recesses preventing a continuity analysis at Low Voltage from extending to the Medium Voltage or Medium Voltage to the High Voltage. As Analysis Result, the Show Flow option is set and you assign color 1 (red) in preview mode (with a redraw can be cleaned) (Fig. 4). 


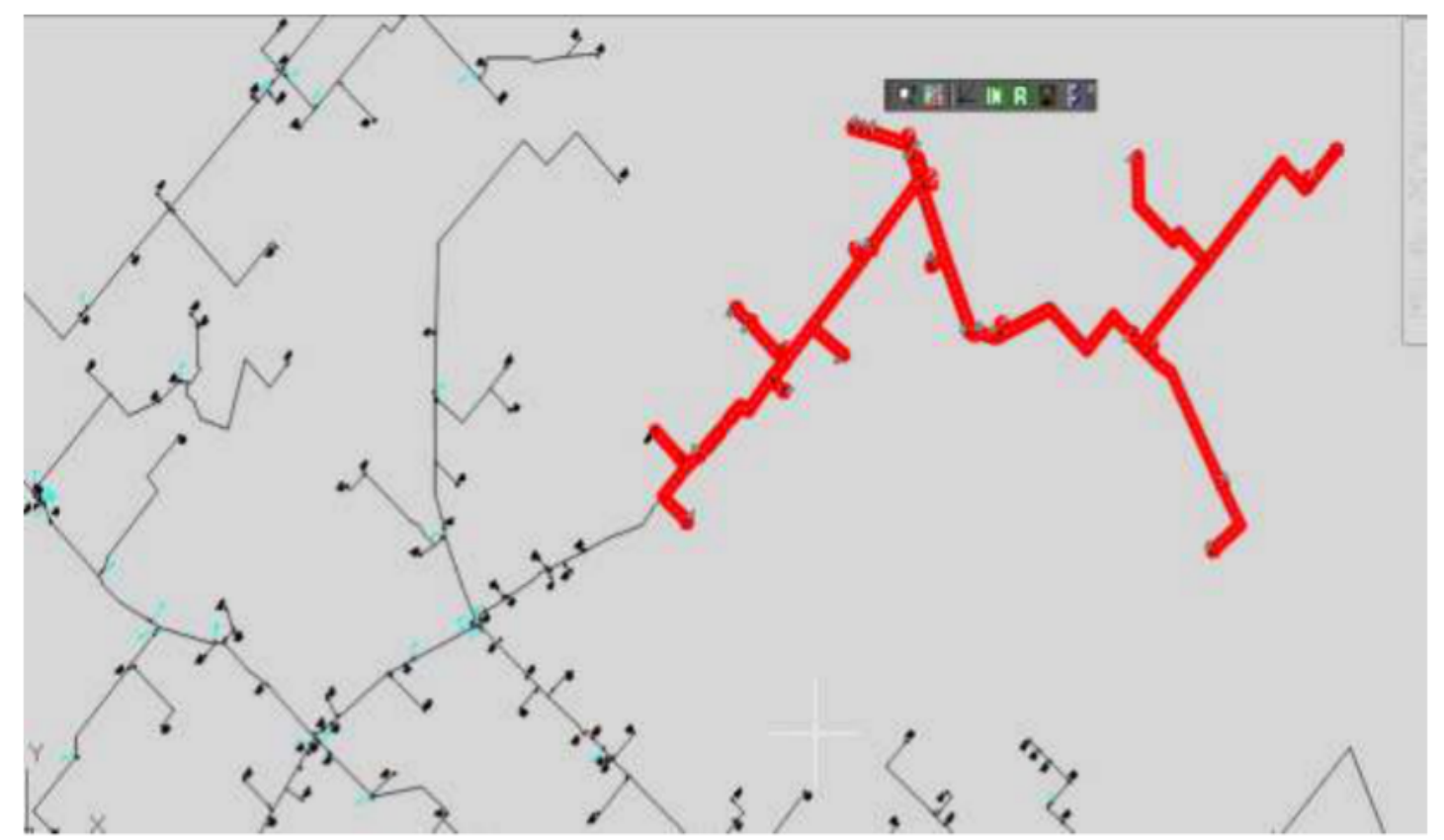

Figure 4 Visualization of continuity analysis

This command does not cause network modifications and is ideal for verifying leg continuity after any network upgrade or maintenance task.

\subsection{Select Network / Customers}

This command (SELNET) allows you to use the continuity analysis result to select the network and/or clients covered.

The operation is similar to that of TESTTOPOLOGIA, but unlike the previous one, as a result it generates a new topology by modifying the thickness and color of the selected legs for quick identification.

The command allows to be used more than once and then use the sum to select the features (network runs, equipment, customer onslaughts and their links to databases).

From this we derive numerous applications of great utility for the technical area of an Electric Power Distributor Company:

- Creating thematic circuit maps: Allows you to view in different colors the network sections and customer rushes for each output of the Transformation Center

- Identification of equipment and transformation centers downstream from a point in the network.

- Identification and selection of customers supplied by Circuit or Transformation Centers to access the database, perform supplied average energy calculations or load reallocation studies.

- Identification of areas affected by interruptions: This utility is the basis of a software that we have called SigreGIS This software implemented in cooperatives in the South East of the Province of Buenos Aires (Balcarce, Mar del Plata, Laguna de Los Padres, San Cayetano ) allows you to identify and store in a "Historical" folder the circuits, equipment and clients affected by each outage along with cause and duration data and then perform quality of service calculations and reports. [7]

Network continuity analysis tools together with the ability to identify and select affected sections has been used in the work

"Identification and Faults Location in Distribution Energy Power Systems from Measurements and Data on GIS Platforms" [9] This computer tool has been developed to 
facilitate the location of faults in medium voltage distributors, based on the measurements of voltage and current of the pre-fault and fault taken at the beginning of a fault distribution. The methodology is based on the analysis of the fault impedance and contemplates the investigation of all the branches in derivation belonging to the affected distributor, with the purpose of contributing to discriminate the faulty section. The response of the calculations is displayed in a graphical interface in a Geographic Information System (GIS) environment where the analysis network is digitized

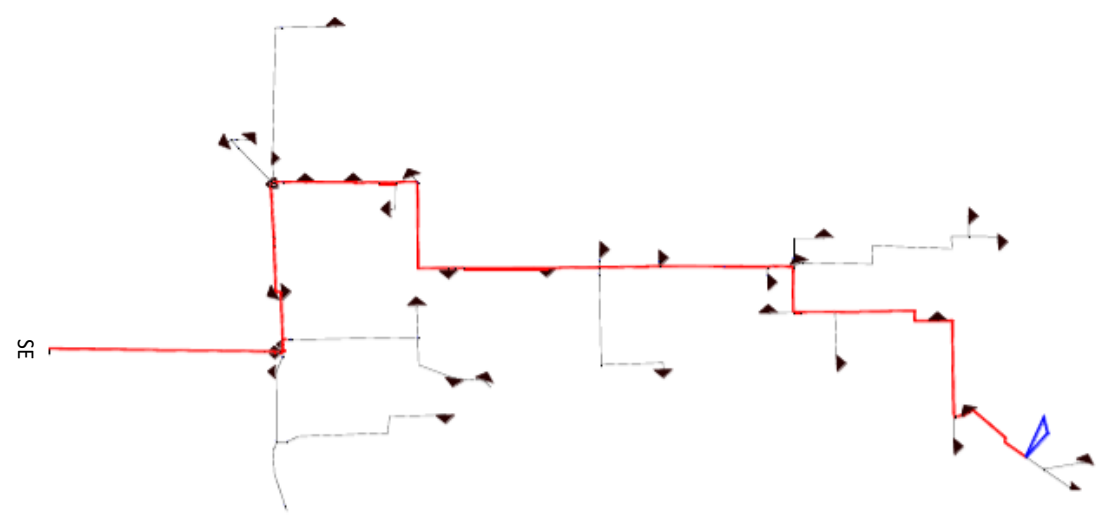

Figure 5. Estimation of single-phase failure. Source: Authors.

\section{CONCLUSION}

From a standardization and specification of the type of feature and layer of the elements digitized according to the function in an electrical network, it is possible to develop tools based on the simple-to-use network topology continuity analyses, which simulate the usual operations in power grids. As a result of these analyses it is possible to view, select and export to be used by calculation software the linked data of each element of the network or customer supplied.

\section{REFERENCES}

[1] S.A. Miloca, N.M.P. Volpi, J. Yuan, C.L.S. Pinto, Expansion planning problem in distribution systems with reliability evaluation: An application in real network using georeferenced database, International Journal of Electrical Power \& Energy Systems, Volume 70, 2015, Pages 9-16,ISSN 0142-0615, https://doi.org/10.1016/j.ijepes.2015.01.004.

[2] Cebrián, J.A. Geographic information and geographic information systems. Santander: DIDOT SA. 1992

[3] Geographic Information Systems (GIS): Basic techniques for biodiversity studies National Node of Information on Biodiversity Spanish Ministry of Economy and Competitiveness, Higher Council for Scientific Research Spain

[4] Autodesk Geospatial White Paper. 2019 Autodesk, Inc.

[5] Bosque Sendra, J. “Sistemas de información geográfica.” Madrid. RIALP.1992

[6] Domínguez, J. Basic concepts and applications of Geographic Information Systems. Technical Desktop Magazine, Marzo/Abril,1994Vol. II, nº 6. p. 28 a 33. Madrid. 
[7] C. Agüero, C. Babic, G. Di Mauro, J. Vignerte Use of the Georeferenced Catastrophe of the D.P.E. for the Location of Faults in Distribution Systems THE 12th LATIN-American Congress On Electricity Generation And Transmission - Clagtee 2017

[8] OCEBA “Normas De Calidad Del Servicio Publico Y Sanciones" Organismo de Control de la Prov. De Buenos Aires.1997

[9] G. di Mauro, C. Agüero, J. Vignerte, D. Anaut, J. Strack. Identification and Faults Location in Distribution Energy Power Systems from Measurements and Data on GIS Platforms. International Journal of Engineering Research \& Science (IJOER) ISSN: 23956992.Vol-2, Issue-6 June- 2016 